PROCEEDINGS OF THE

AMERICAN MATHEMATICAL SOCIETY

Volume 138, Number 2, February 2010, Pages 467-480

S 0002-9939(09)10175-2

Article electronically published on October 6, 2009

\title{
ZEROS OF SOME LEVEL 2 EISENSTEIN SERIES
}

\author{
SHARON GARTHWAITE, LING LONG, HOLLY SWISHER, AND STEPHANIE TRENEER
}

\author{
(Communicated by Ken Ono)
}

\begin{abstract}
The zeros of classical Eisenstein series satisfy many intriguing properties. Work of F. Rankin and Swinnerton-Dyer pinpoints their location to a certain arc of the fundamental domain, and recent work by Nozaki explores their interlacing property. In this paper we extend these distribution properties to a particular family of Eisenstein series on $\Gamma(2)$ because of its elegant connection to a classical Jacobi elliptic function $c n(u)$ which satisfies a differential equation. As part of this study we recursively define a sequence of polynomials from the differential equation mentioned above that allows us to calculate zeros of these Eisenstein series. We end with a result linking the zeros of these Eisenstein series to an $L$-series.
\end{abstract}

\section{INTRODUCTION}

Modular forms are functions defined on the complex upper half-plane $\mathbb{H}$ that transform in a nice way with respect to subgroups of $\mathrm{SL}_{2}(\mathbb{Z})$. These functions have proved to be intricately related to deep results in many areas of number theory, combinatorics, algebraic geometry, and other areas of mathematics (cf. Ono04). For example, modular forms are deeply involved in the proof of Fermat's last theorem, results in partition theory, and denominator formulas for the monster group. Eisenstein series are primary components of modular forms. That is, the weighted algebra of all integral weight holomorphic modular forms for $\mathrm{SL}_{2}(\mathbb{Z})$ is generated by $E_{4}(z)$ and $E_{6}(z)$, the unique normalized Eisenstein series of weights 4 and 6 for $\mathrm{SL}_{2}(\mathbb{Z})$, respectively. A nice generating function of the classical Eisenstein series $E_{2 k}(z)$ is the Weierstrass $\wp$ function, which is an elliptic function satisfying

$$
\left(\wp^{\prime}(u)\right)^{2}=4 \wp(u)^{3}-a_{4} \wp(u)-a_{6},
$$

where $a_{4}, a_{6}$ are scalar multiples of $E_{4}$ and $E_{6}$, respectively.

Location of the zeros of Eisenstein series is of fundamental importance. Provided with such information, Li, Long, and Yang construct noncongruence cusp forms from congruence Eisenstein series LLY05. Additionally, in a paper of Ono and Papanikolas OP04, an interesting formula is highlighted which relates the location of the zeros of $E_{2 k}(z)$ to special values of the Riemann zeta function $\zeta(s)$ :

$$
\frac{2}{\zeta(1-2 k)}=60 \cdot 2 k-\sum_{\tau \in \mathbb{H} / S L_{2}(\mathbb{Z})} e_{\tau} \operatorname{ord}_{\tau}\left(E_{2 k}(\tau)\right) j(\tau),
$$

Received by the editors June 4, 2009

2000 Mathematics Subject Classification. Primary 11F11; Secondary 11F03.

The second author was supported in part by the NSA grant no. H98230-08-1-0076.

(C)2009 American Mathematical Society 
where $e_{i}=1 / 2, e_{\frac{-1+\sqrt{-3}}{2}}=1 / 3$, and $e_{\tau}=1$ for nonelliptic points, $\tau$.

In RSD70, F.K.C. Rankin and H.P.F. Swinnerton-Dyer study the location of the zeros of $E_{2 k}(z)$. By using an elementary and elegant argument, they demonstrate that all zeros of $E_{2 k}(z)$ inside the fundamental domain for $S L_{2}(\mathbb{Z})$ are located on the arc $\left\{z=e^{i \theta}, \pi / 2 \leq \theta \leq 2 \pi / 3\right\}$. Equivalently, the $j$-values of these zeros are all real and are within the interval $[0,1728]$, where $j(z)$ is the well-known classical modular function which parametrizes isomorphism classes of elliptic curves. In [Koh04], Kohnen gives an explicit formula for the zeros of $E_{2 k}(z)$.

Recently, there have been several results generalizing [RSD70] to Eisenstein series of other groups [Hah07, MNS07, Shi07, etc.], other modular forms [Get04, Gun06], and certain weakly holomorphic modular forms DJ08. Moreover, Nozaki proves that the zeros of the classical Eisenstein series $E_{2 k}(z)$ interlace with the zeros of $E_{2 k+12}(z)$ Noz08. Despite these results, many mysteries remain about Eisenstein series and their zeros. For example, Gekeler studies polynomials $\varphi_{2 k}(x)$ which encode the $j$-values of the nonelliptic zeros of $E_{2 k}(z)$ Gek01. He conjectures that the $\varphi_{2 k}(x)$ are irreducible with Galois group $S_{d}$, where $d$ is the degree of $\varphi_{2 k}(x)$.

In Section 2 of this paper, we define a series of odd-weight Eisenstein series denoted by $G_{2 k+1}(z)$ for $\Gamma(2)$, the principal level 2 congruence subgroup. In LY05], Long and Yang use $G_{2 k+1}(z)$ to give a second proof to some beautiful formulae of Milne Mil02 on representing natural numbers in terms of sums of squares or triangular numbers. Our attention was drawn to this family of Eisenstein series $G_{2 k+1}(z)$ largely because it has an elegant generating function $c n(u)$, a classical Jacobi elliptic function Han58 satisfying

$$
\left(\frac{\mathrm{d} c n(u)}{\mathrm{d} u}\right)^{2}=\left(1-c n^{2}(u)\right)\left(1-\lambda+\lambda c n^{2}(u)\right) .
$$

Here $\lambda$ is the classical lambda function which parameterizes all isomorphism classes of elliptic curves with full 2-torsion structure. The equation (1.2) gives rise to recursions satisfied by $G_{2 k+1}(z)$. Consequently, one can compute the $\lambda$-values of the zeros of $G_{2 k+1}(z)$ with great convenience and ease. Data from the first few dozen $k$-values indicates that all $\lambda$-values of the zeros of $G_{2 k+1}(z)$ are real and are within the interval $(-\infty, 0]$. Moreover, this data indicates that the zeros of $G_{2 k-1}(z)$ interlace with the zeros of $G_{2 k+1}(z)$.

In Section 3 we investigate the location of the zeros of $G_{2 k+1}(z)$ within the following fixed fundamental domain of $\Gamma(2)$ (cf. Figure 1 in Section 2):

$$
D=\{z \in \mathbb{H}:-1 \leq \operatorname{Re}(z) \leq 1,|z-1 / 2| \geq 1 / 2, \quad|z+1 / 2| \geq 1 / 2\} .
$$

A direct application of the argument of Rankin and Swinnerton-Dyer shows that at least one third of the zeros of $G_{2 k+1}(z)$ lie in

$$
\{z \in \mathbb{H}:|z+1 / 2|=1 / 2\}
$$

or, equivalently, have $\lambda$-values in $(-\infty, 0]$. By refining this argument, we will show the following improvement.

Theorem 1.1. For $k \geq 1$, at least $90 \%$ of the zeros of $G_{2 k+1}(z)$ have real $\lambda$-values in the range $(-\infty, 0]$.

In addition, by restricting our domain slightly we obtain the following result about the separation property of the zeros of $G_{2 k-1}(z)$ and $G_{2 k+1}(z)$ following an approach of Nozaki Noz08]. We state this result in terms of the related function 
$F_{2 k+1}\left(z_{\theta}\right)$ defined in (3.1), where $z_{\theta}:=\frac{1}{2} e^{i \theta}-\frac{1}{2}$. Before stating the result, we define for an integer $k>15$ the intervals

$$
I_{j, 2 k-1}=\left(\alpha_{j, k}-\frac{2 \pi}{(2 k+1)(2 k-1)}, \alpha_{j, k}+\frac{2 \pi}{(2 k+1)(2 k-1)}\right)
$$

for each $j=1, \ldots, k-1$, where $\alpha_{j, k}:=2 \pi j /(2 k-1)$. Furthermore, let

$$
I_{2 k-1}:=\bigcup_{j} I_{j, 2 k-1} \text {. }
$$

Theorem 1.2. Let $k>15$ be an integer, and let $I_{j, 2 k-1}, I_{2 k-1}$ be as defined above. Then the zeros of $F_{2 k-1}\left(z_{\theta}\right)$ and $F_{2 k+1}\left(z_{\theta}\right)$ in $[\pi / 10,9 \pi / 10]$ are restricted to $I_{2 k-1}$ and $I_{2 k+1}$ respectively (in fact each $I_{j, 2 k-1}$ and $I_{j, 2 k+1}$ in $[\pi / 10,9 \pi / 10]$ contains an odd number of zeros). Moreover, the intervals are pairwise disjoint, and the $I_{j, 2 k-1}$ are separated by the $I_{j, 2 k+1}$.

In Section 4 we obtain a formula (Corollary 4.2) similar to equation (1.1) which relates the sum of the $\lambda$-values of the zeros of $G_{2 k+1}(z)$ to the special values of an explicit $L$-series.

We note that the properties of our Eisenstein series $G_{2 k+1}(z)$ are similar to other families of level 2 Eisenstein series studied by Tim Huber to which we expect our results can be extended. Furthermore, the behavior of our Eisenstein series is similar to all other known cases for congruence subgroups of genus zero with relatively simple fundamental domains. It would be very interesting to know what to expect for Eisenstein series or cusp forms that are invariant under congruence subgroups with more complex fundamental domains or higher genus.

The authors would like to acknowledge that this project and collaboration was initiated at the Women in Numbers Conference at BIRS. The authors thank the organizers Kristen Lauter, Rachel Pries and Renate Scheidler, along with BIRS, the Fields Institute, Microsoft Research, the NSA, PIMS, and the University of Calgary for their generous support of this conference.

\section{2. $\Gamma(2)$ AND ITS MODULAR FORMS}

Let $\Gamma(2)$ be the principal level 2 congruence subgroup consisting of matrices which become the identity under the natural modulo 2 homomorphism. This group is of genus 0 and has a fundamental domain shown in Figure 1. Let $q=e^{2 \pi i z}$; the

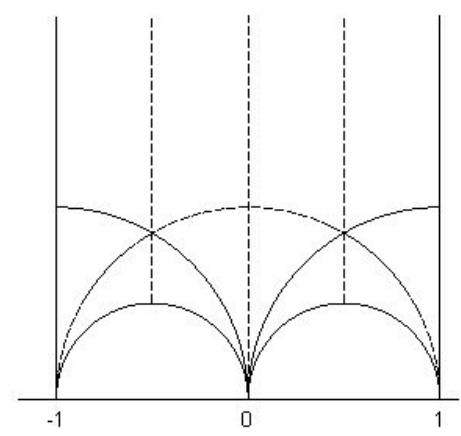

Figure 1. A fundamental domain for $\Gamma(2)$ 
Jacobi theta functions are defined by

$$
\Theta_{2}(z):=q^{1 / 8} \sum_{n \in \mathbb{Z}} q^{n(n+1) / 2}, \Theta_{3}(z):=\sum_{n \in \mathbb{Z}} q^{n^{2} / 2}, \text { and } \Theta_{4}(z):=\sum_{n \in \mathbb{Z}}(-1)^{n} q^{n^{2} / 2} .
$$

The square of each of the above series is a weight 1 modular form for $\Gamma(2)$. The classical lambda function is defined as $\lambda(z):=\frac{\Theta_{2}^{4}(z)}{\Theta_{3}^{4}(z)}$, and it generates the field of meromorphic modular functions for $\Gamma(2)$.

2.1. Some odd weight level 2 Eisenstein series. We now focus on a particular family of Eisenstein series of odd weight on $\Gamma(2)$ investigated in [LLY05]. To begin, we define the character

$$
\chi(\gamma):=\chi(d)=\left(\frac{-1}{d}\right),
$$

where $\gamma=\left(\begin{array}{ll}a & b \\ c & d\end{array}\right)$ and $\left(\frac{-1}{\bullet}\right)$ denotes a Legendre symbol. For $k \geq 1$, we define

$$
E_{2 k+1, \chi}(z):=\frac{1}{2} \sum_{\substack{(c, d) \equiv(0,1)(2) \\(c, d)=1}} \chi(d) \cdot(c z+d)^{-(2 k+1)}
$$

and recall some results about $E_{2 k+1, \chi}(z)$ (see [LLY05, Lemmas $\left.5.2-5.5\right]$ ).

Lemma 2.1. Define the nth Euler number $e_{n}$ by $\sec (t)=\sum_{n=0}^{\infty} \frac{e_{n}}{n !} t^{n}$. The function $E_{2 k+1, \chi}(z)$ is a modular form of weight $2 k+1$ on $\Gamma(2)$ with Fourier expansion at $\infty$ given by

$$
E_{2 k+1, \chi}(z)=1+\frac{4(-1)^{k}}{e_{2 k}} \sum_{r=1}^{\infty} \chi(r) \frac{r^{2 k} q^{r / 2}}{1-q^{r / 2}}
$$

For any positive integer $k$ let

$$
S_{k}(\alpha, \beta)=S_{k}(\alpha, \beta)(z):=\sum_{\substack{(c, d) \equiv(\alpha, \beta)(4) \\(c, d)=1}} \frac{1}{(c z+d)^{k}} .
$$

By the explicit description of $\chi$,

$$
E_{2 k+1, \chi}(z)=\frac{1}{2}\left(S_{2 k+1}(0,1)+S_{2 k+1}(2,1)-S_{2 k+1}(0,3)-S_{2 k+1}(2,3)\right) .
$$

Recall that if $f$ is a meromorphic function on $\mathbb{H}, \gamma=\left(\begin{array}{ll}a & b \\ c & d\end{array}\right) \in \mathrm{SL}_{2}(\mathbb{Z})$, and $k$ is an integer, then the slash operator in weight $k$ is

$$
\left.f(z)\right|_{k} \gamma:=(c z+d)^{-k} f(\gamma z)
$$

We use $\left.f\right|_{\gamma}$ to denote $\left.f(z)\right|_{k} \gamma$. The following lemma for odd $k$ follows analogously from Lemma 3.3 in [LLY05].

Lemma 2.2. If $\gamma \in \mathrm{SL}_{2}(\mathbb{Z})$ and $S_{k}$ is defined as above for odd $k \geq 1$, then

$$
\left.\left.S_{k}(\alpha, \beta)\right|_{\gamma}=S_{k}((\alpha, \beta) \cdot \gamma) \quad(\bmod 4)\right),
$$

where $(\alpha, \beta) \cdot \gamma(\bmod 4)$ denotes matrix multiplication modulo 4. 
2.2. A generating function. Recall that $c n(u)$ is a classical Jacobi elliptic function which satisfies (cf. Han58, p. 256])

$$
\frac{\kappa K}{2 \pi} c n\left(\frac{2 K u}{\pi}\right)=\frac{\sqrt{q} \cos u}{1+q}+\frac{\sqrt{q^{3}} \cos 3 u}{1+q^{3}}+\frac{\sqrt{q^{5}} \cos 5 u}{1+q^{5}}+\cdots,
$$

where $\sqrt{\kappa}=\frac{\Theta_{2}(2 z)}{\Theta_{3}(2 z)}$ (cf. [Han58, p. 241]) and hence $\lambda(2 z)=\kappa^{2}$ and $K=\frac{\pi}{2} \Theta_{3}^{2}(2 z)$. For the remainder of this section we simply write $\lambda$ for $\lambda(2 z)$. To see how the family of Eisenstein series $E_{2 k+1, \chi}$ relates to $\mathrm{cn}(u)$, we consider the expansion of $E_{2 k+1, \chi}$ at the cusp 1, given by $\left.E_{2 k+1, \chi}\right|_{\gamma_{0}}$, where $\gamma_{0}=\left(\begin{array}{cc}1 & -1 \\ 1 & 0\end{array}\right)$. The previous lemma allows us to easily calculate $\left.E_{2 k+1, \chi}\right|_{\gamma_{0}}$ in terms of the $S_{k}$, which brings us to the following conclusion:

$$
\left.E_{2 k+1, \chi}\right|_{\gamma_{0}}(z)=\frac{1}{2}\left(S_{2 k+1}(1,0)+S_{2 k+1}(3,2)-S_{2 k+1}(3,0)-S_{2 k+1}(1,2)\right) .
$$

It is this function $G_{2 k+1}(z):=\left.E_{2 k+1, \chi}\right|_{\gamma_{0}}(z)$, whose zeros we study. It has been shown ([LY05, Lemma 5.5) that

$$
G_{2 k+1}(z)=\frac{4(-i)^{2 k+1}}{e_{2 k}} \sum_{r=1}^{\infty} \frac{(2 r-1)^{2 k} q^{(2 r-1) / 4}}{1+q^{(2 r-1) / 2}} .
$$

In addition, we define $G_{1}(z)=-i \Theta_{2}^{2}(z)$ (cf. [LY05, Lemma 5.4).

Using the series expansion $\cos (x)=\sum_{n=0}^{\infty}(-1)^{n} \frac{x^{2 n}}{(2 n) !}$, we have

$$
\begin{aligned}
\frac{\kappa K}{2 \pi} c n\left(\frac{2 K u}{\pi}\right) & =\sum_{k=0}^{\infty} \frac{(-1)^{k}}{(2 k) !}\left(\sum_{r=1}^{\infty} \frac{(2 r-1)^{2 k} q^{(2 r-1) / 2}}{1+q^{2 r-1}}\right) u^{2 k} \\
& =\sum_{k=0}^{\infty} \frac{i e_{2 k}}{4(2 k) !} G_{2 k+1}(2 z) u^{2 k} .
\end{aligned}
$$

As an immediate consequence, we have the following proposition.

Proposition 2.3. Let $\kappa=\frac{\Theta_{2}^{2}(2 z)}{\Theta_{3}^{2}(2 z)}$ and $K=\frac{\pi}{2} \Theta_{3}^{2}(2 z)$. We have that

$$
c n(u)=\sum_{k=0}^{\infty} \frac{i e_{2 k} \pi^{2 k+1}}{2^{2 k+1}(2 k) !} \frac{G_{2 k+1}(2 z)}{K^{2 k+1} \kappa} u^{2 k} .
$$

From $c n(u)$ satisfying (1.2) (cf. Han58, p. 247]), we have that

$$
\operatorname{cn}(u)=1-\frac{u^{2}}{2 !}+(1+4 \lambda) \frac{u^{4}}{4 !}-\left(1+44 \lambda+16 \lambda^{2}\right) \frac{u^{6}}{6 !}+\cdots .
$$

Note that the choice $K=\frac{\pi}{2} \Theta_{3}^{2}(2 z)$ aligns the constant terms of equations (2.3) and (2.4). We have that

$$
\frac{(-1)^{k} i \pi^{2 k+1} e_{2 k}}{2^{2 k+1}} \cdot \frac{G_{2 k+1}(2 z)}{K^{2 k+1} \kappa}=\frac{(-1)^{k} i e_{2 k} G_{2 k+1}(2 z)}{\Theta_{3}^{4 k}(2 z) \Theta_{2}^{2}(2 z)}=\frac{(-1)^{k} e_{2 k} G_{2 k+1}(2 z)}{\Theta_{3}^{4 k}(2 z) G_{1}(2 z)}
$$

is a modular function for $\Gamma(2)$ which is holomorphic everywhere except possibly a pole of finite order at the cusp infinity coming from $\Theta_{3}^{4 k}(2 z)$. Consequently, for $k \geq 1$ it is a degree $(k-1)$ polynomial in terms of $\lambda$, denoted $p_{2 k+1}(\lambda)$, having the 
same zeros as $G_{2 k+1}$ in the fundamental domain. We list the first six below:

$$
\begin{array}{ll}
p_{1}(\lambda)=1, & p_{7}(\lambda)=1+44 \lambda+16 \lambda^{2}, \\
p_{3}(\lambda)=1, & p_{9}(\lambda)=1+408 \lambda+912 \lambda^{2}+64 \lambda^{3}, \\
p_{5}(\lambda)=1+4 \lambda, & p_{11}(\lambda)=1+3688 \lambda+30764 \lambda^{2}+15808 \lambda^{3}+256 \lambda^{4} .
\end{array}
$$

There are $k-1$ nontrivial zeros counting multiplicity, in addition to the trivial zeros of $G_{2 k+1}$ at the cusps. Calculations for $2 k+1 \leq 51$ reveal some striking numerical trends. The $\lambda$-values of the zeros of $G_{2 k+1}$ all lie in $(-\infty, 0)$. We list the numerical zeros of the first few $p_{2 k+1}(\lambda)$ below.

\begin{tabular}{|c|c||c|c|}
\hline$k=2$ & $\{-0.25\}$ & $k=4$ & $\{-0.0025,-0.4598,-13.788\}$ \\
\hline$k=3$ & $\{-.0229,-2.7271\}$ & $k=5$ & $\{-.00027,-.1280,-1.8792,-59.7425\}$ \\
\hline
\end{tabular}

Moreover, the $\lambda$-zeros of $p_{2 k-1}(\lambda)$ interlace with the $\lambda$-zeros of $p_{2 k+1}(\lambda)$. In addition, the polynomials $p_{2 k+1}(\lambda)$ are irreducible with Galois group $S_{k-1}$ for $k \leq 9$.

\section{Locating The ZERos of $G_{2 k+1}(z)$}

Based on the numerical evidence above, we now turn our focus to the $\lambda$-values of the zeros of $G_{2 k+1}(z)$. Proving that the $\lambda$-values lie in $(-\infty, 0]$ is equivalent to proving that the zeros of $G_{2 k+1}(z)$ lie on the line $\operatorname{Re}(z)=1$, a boundary arc of the fundamental domain for $\Gamma(2)$. This, in turn, is equivalent to proving that the zeros of $G_{2 k+1} \mid \gamma_{1}(z)$, where $\gamma_{1}=\left(\begin{array}{cc}0 & -1 \\ 1 & 0\end{array}\right)$, lie on the arc $\left|z+\frac{1}{2}\right|=\frac{1}{2}$, as we mentioned in Section 1. Our main strategy is to generalize the approach of Rankin and Swinnerton-Dyer. In particular, we will need to refine estimates of the terms arising in this approximation.

We first find, using Lemma 2.2, that

$$
\left.G_{2 k+1}\right|_{\gamma_{1}}(z)=\frac{1}{2}\left(S_{2 k+1}(0,3)+S_{2 k+1}(2,1)-S_{2 k+1}(0,1)-S_{2 k+1}(2,3)\right) .
$$

We now wish to count the number of zeros of $\left.G_{2 k+1}\right|_{\gamma_{1}}(z)$ that must lie on the $\operatorname{arc}\left|z+\frac{1}{2}\right|=\frac{1}{2}$.

Lemma 3.1. Let $\alpha$ be even and $z_{\theta}=\frac{1}{2} e^{i \theta}-\frac{1}{2}$. Then

$$
S_{k}(\alpha, \beta)\left(z_{\theta}\right)=S_{k}\left(\frac{\alpha}{2}, \beta-\frac{\alpha}{2}\right)\left(e^{i \theta}\right)+S_{k}\left(\frac{\alpha}{2}+2, \beta-2-\frac{\alpha}{2}\right)\left(e^{i \theta}\right) .
$$

Proof. The lemma follows by separating the terms in $S_{k}(\alpha, \beta)\left(z_{\theta}\right)$ for pairs $(c, d)$ according to the two possible values of $\frac{c}{2}$ modulo 4 and by the fact that $(c, d)=1$ implies $(c, d)=\left(\frac{c}{2}, d-\frac{c}{2}\right)$.

By Lemma 3.1 and the observation that $S_{k}(-a,-b)=-S_{k}(a, b)$, we have

$$
\left.G_{2 k+1}\right|_{\gamma_{1}}\left(z_{\theta}\right)=\left(S_{2 k+1}(0,3)+S_{2 k+1}(1,0)+S_{2 k+1}(2,1)+S_{2 k+1}(3,2)\right)\left(e^{i \theta}\right) .
$$

We next factor out $\left(e^{\frac{i \theta}{2}}\right)^{-2 k-1}$ following RSD70 and define

$$
\begin{aligned}
F_{2 k+1}\left(z_{\theta}\right): & =\left.\left(e^{\frac{-i \theta}{2}}\right)^{-2 k-1} G_{2 k+1}\right|_{\gamma_{1}}\left(z_{\theta}\right) \\
& =\left(\widetilde{S}_{2 k+1}(0,3)+\widetilde{S}_{2 k+1}(1,0)+\widetilde{S}_{2 k+1}(2,1)+\widetilde{S}_{2 k+1}(3,2)\right)(\theta),
\end{aligned}
$$


where

$$
\widetilde{S}_{k}(\alpha, \beta)(\theta):=\sum_{\substack{(c, d) \equiv(\alpha, \beta)(4) \\(c, d)=1}}\left(c e^{i \theta / 2}+d e^{-i \theta / 2}\right)^{-k} .
$$

From equation (3.1) we see that $F_{2 k+1}\left(z_{\theta}\right)$ is purely imaginary by noting that $F_{2 k+1}\left(\bar{z}_{\theta}\right)=-F_{2 k+1}\left(z_{\theta}\right)$, as conjugation interchanges each pair of sums. Moreover, in each sum, $c$ and $d$ have opposite parity.

A direct application of the analysis in [RSD70] shows there are exactly the right number of zeros when $\theta$ ranges through $\left[\frac{\pi}{3}, \frac{2 \pi}{3}\right]$. In other words, one third of the zeros are expected to be on the above arc with $\theta$ in $\left[\frac{\pi}{3}, \frac{2 \pi}{3}\right]$. The real difficulty comes from the analysis when $\theta$ is close to 0 or $\pi$.

3.1. Extraction of the main term. To begin our analysis of $\left.G_{2 k+1}\right|_{\gamma_{1}}\left(z_{\theta}\right)$ on the $\operatorname{arc}\left|z+\frac{1}{2}\right|=\frac{1}{2}$, we first extract the two terms for which $c^{2}+d^{2}=1$ (one occurring in each of $\widetilde{S}_{2 k+1}(0,3)$ and $\left.\widetilde{S}_{2 k+1}(1,0)\right)$ to create our main term,

$$
\left(e^{\frac{i \theta}{2}}\right)^{-2 k-1}-\left(e^{\frac{-i \theta}{2}}\right)^{-2 k-1}=-2 i \sin \left(\frac{\theta(2 k+1)}{2}\right) .
$$

Thus, we have

$$
F_{2 k+1}\left(z_{\theta}\right)=-2 i \sin \left(\frac{\theta(2 k+1)}{2}\right)+R_{2 k+1}\left(z_{\theta}\right),
$$

where $R_{2 k+1}\left(z_{\theta}\right)$ or simply $R\left(z_{\theta}\right)$ is purely imaginary and denotes the error term obtained by summing over all remaining terms.

3.2. Bounding the error term. We now turn to bounding $R\left(z_{\theta}\right)$. Here we will consider the contribution of terms satisfying $c^{2}+d^{2}=N$, for $N>1$. We take advantage of the symmetry in our remaining terms and define the following partial sum. For each ordered pair of nonnegative integers $(a, b)$ chosen such that $a$ is odd and $b$ is even to avoid double-counting, define

$$
P(a, b)(\theta)=\sum_{(|c|,|d|)=(a, b) \text { or }(b, a)}\left(c e^{i \theta / 2}+d e^{-i \theta / 2}\right)^{-2 k-1},
$$

where the sum contains only terms occurring in $F_{2 k+1}\left(z_{\theta}\right)$. For example,

$$
P(3,0)=\left(\left(-3 e^{i \theta / 2}\right)^{-2 k-1}+\left(3 e^{-i \theta / 2}\right)^{-2 k-1}\right)=\frac{2 i}{3^{2 k+1}} \sin \left(\frac{\theta(2 k+1)}{2}\right) .
$$

Due to symmetry, each partial sum $P(a, b)$ is purely imaginary as before.

3.3. The case when $N \leq 100$. We now give upper bounds on the terms for which $1<N \leq 100$. We assume that $2 k+1>51$ as we have numerically verified the location of zeros for low-weight cases.

When $b=0$ we have the following cases:

$$
(a, b) \in\{(3,0),(5,0),(7,0),(9,0)\} .
$$

Here,

$$
|P(a, b)(\theta)|=\left|\frac{2}{a^{2 k+1}} \sin \left(\frac{(2 k+1) \theta}{2}\right)\right| \leq \frac{2}{a^{51}} .
$$

The contribution from these terms to the error term is smaller than

$$
E_{1}=\sum_{n=1}^{4} \frac{2}{(2 n+1)^{51}}<10^{-24} .
$$


When $b \neq 0$, then for each term in $P(a, b)$ we have

$$
\left|c e^{i \theta / 2}+d e^{-i \theta / 2}\right|=\left|a e^{i \theta / 2} \pm b e^{-i \theta / 2}\right| \text { or }\left|b e^{i \theta / 2} \pm a e^{-i \theta / 2}\right| .
$$

In the first case,

$$
\begin{aligned}
\left|a e^{i \theta / 2} \pm b e^{-i \theta / 2}\right|^{-2 k-1} & =|(a \pm b) \cos (\theta / 2)+i(a \mp b) \sin (\theta / 2)|^{-2 k-1} \\
& = \begin{cases}\left((a-b)^{2}+4 a b \cos ^{2}(\theta / 2)\right)^{-k-1 / 2} & \text { if }+, \\
\left((a-b)^{2}+4 a b \sin ^{2}(\theta / 2)\right)^{-k-1 / 2} & \text { if }-.\end{cases}
\end{aligned}
$$

By symmetry, the second case yields the same result. When $a$ and $b$ are nonzero, $P(a, b)$ contains four terms, two of each type in (3.4). Therefore,

$$
|P(a, b)| \leq \frac{2}{\left((a-b)^{2}+4 a b \sin ^{2}(\theta / 2)\right)^{k}}+\frac{2}{\left((a-b)^{2}+4 a b \cos ^{2}(\theta / 2)\right)^{k}} .
$$

Note that

$$
\max \left(\cos ^{2}(\theta / 2), \sin ^{2}(\theta / 2)\right) \geq 1 / 2 .
$$

Moreover, if we limit our choice of $\theta$ to the interval $(0.05 \pi, 0.95 \pi)$, then

$$
\min \left(\cos ^{2}(\theta / 2), \sin ^{2}(\theta / 2)\right)>(.079)^{2} .
$$

Hence we conclude that when $b$ is nonzero,

$$
|P(a, b)| \leq \frac{2}{\left((a-b)^{2}+4 a b(.079)^{2}\right)^{k}}+\frac{2}{\left((a-b)^{2}+2 a b\right)^{k}} .
$$

Now consider the cases

$$
(a, b) \in\{(1,2),(3,2),(3,4),(5,4),(5,6),(7,6)\},
$$

with $|a-b|=1$. Summing the bounds in (3.5) for the six pairs $(a, b)$ listed above yields that the contribution from these terms is smaller than

$$
E_{2}=0.656 \text {. }
$$

The remaining cases with $N \leq 100$ are

$$
(a, b) \in\{(1,4),(1,6),(1,8),(3,6),(3,8),(5,2),(5,8),(7,2),(7,4),(9,2),(9,4)\} .
$$

For these we note that $|a-b| \geq 3$. Reasoning as above yields

$$
|P(a, b)|<\frac{4}{9^{k}} .
$$

Hence, the total contribution to the error term is bounded by

$$
E_{3}=11 \cdot \frac{4}{9^{k}} \leq \frac{4}{9^{25}}<10^{-10} .
$$

In total, we have

$$
\left|\sum_{a^{2}+b^{2} \leq 100} P(a, b)\right| \leq E_{1}+E_{2}+E_{3}<0.657 .
$$


3.4. The case when $N>100$. We now consider terms $c^{2}+d^{2}=N$ with $N \geq 101$, again assuming that $2 k+1>51$. The number of terms satisfying $c^{2}+d^{2}=N$ is at most $2\left(2 N^{1 / 2}+1\right) \leq 5 N^{1 / 2}$. Note that

$$
\left|c e^{i \theta / 2}+d e^{-i \theta / 2}\right|^{2}=c^{2}+2 c d \cos \theta+d^{2} .
$$

If we restrict ourselves to $\theta$-values with $|\cos \theta| \leq \alpha$, for some $\alpha \in(0,1)$, then

$$
c^{2}+2 c d \cos \theta+d^{2} \geq(1-\alpha)\left(c^{2}+d^{2}\right) .
$$

Thus, we have

$$
\left|R\left(z_{\theta}\right)\right|<0.657+\sum_{N=101}^{\infty} 5 N^{1 / 2}((1-\alpha) N)^{-k-1 / 2} .
$$

By bounding this latter sum with an appropriate integral, we have

$$
\left|R\left(z_{\theta}\right)\right|<0.657+(1-\alpha)^{-k-\frac{1}{2}}\left(\frac{5}{k-1} \cdot 100^{-k+1}\right) .
$$

We pick $\alpha=.9877$, which corresponds to $\theta \in(0.05 \pi, 0.95 \pi)$. Then $\left|R\left(z_{\theta}\right)\right|<2$.

3.5. Proof of Theorem 1.1. Following [RSD70, we consider the values $\theta$ for which $2 \sin \left(\frac{\theta(2 k+1)}{2}\right)= \pm 2$ and then apply the Intermediate Value Theorem to the function $i F_{2 k+1}\left(z_{\theta}\right)$. In doing so we conclude that this function, and hence also $G_{2 k+1}||_{\gamma_{1}}\left(z_{\theta}\right)$, must have at least one zero in each interval of the form

$$
\left[\frac{A \pi}{2 k+1}, \frac{(A+2) \pi}{2 k+1}\right] \subset(0.05 \pi, 0.95 \pi),
$$

where $A$ is an odd positive integer. We also recall that $G_{2 k+1}$ has altogether $k-1$ nontrivial zeros. Thus, having previously considered $2 k+1 \leq 51$ computationally, we can now verify that at least $90 \%$ of the zeros of $G_{2 k+1}(z)$ do indeed lie on the boundary $\operatorname{Re}(z)=1$ and hence have real $\lambda$-values in $(-\infty, 0]$. This concludes the proof of Theorem 1.1 .

3.6. Proof of Theorem 1.2, Now that we have pinpointed the location of the zeros of $G_{2 k+1}(z)$, we turn our attention to the relationship between the zeros of $G_{2 k-1}(z)$ and $G_{2 k+1}(z)$. Numerical evidence suggests that the zeros of $F_{2 k-1}\left(z_{\theta}\right)$ and $F_{2 k+1}\left(z_{\theta}\right)$, the shifted Eisenstein series, interlace in the same manner as demonstrated by Nozaki for the classical case with $E_{k}(z)$ and $E_{k+12}(z)$. We now follow Nozaki's strategy by relating the zeros of $F_{2 k+1}\left(z_{\theta}\right)$ to the zeros of the main term of this series, a well-understood trigonometric function with regularly spaced zeros. We then show that the additional error terms for the Eisenstein series will not cause the zeros of $F_{2 k+1}\left(z_{\theta}\right)$ to stray far from the zeros of the main term.

We must first strengthen the bounds on the error estimates given in the previous section. In order to do this we will need to change our lower bound on $\sin (\theta / 2)$ and $\cos (\theta / 2)$ near the cusps $\theta=0, \pi$. Thus, we narrow our focus to theta values in the range $[\pi / 10,9 \pi / 10]$.

Recall that the main term of $F_{2 k+1}\left(z_{\theta}\right)$ given in (3.2) is

$$
f_{2 k+1}(\theta):=-2 i \sin \left(\frac{\theta(2 k+1)}{2}\right) \text {. }
$$


Let $\alpha_{j, k}$ denote the zeros of $f_{2 k-1}$ on $(0, \pi)$, that is,

$$
\alpha_{j, k}=\frac{2 \pi j}{2 k-1}, j=1, \ldots, k-1 .
$$

Note that $\alpha_{j, k+1}<\alpha_{j, k}<\alpha_{j+1, k+1}$ for $j=1, \ldots, k-1$, and

$$
\frac{1}{2} \min _{j=1, \ldots, k-1}\left\{\alpha_{j}-\beta_{j}, \beta_{j+1}-\alpha_{j}\right\}=\frac{2 \pi}{(2 k-1)(2 k+1)} .
$$

Our aim is to show that the zeros of $F_{2 k-1}\left(z_{\theta}\right)$ and $F_{2 k+1}\left(z_{\theta}\right)$ are within this distance of $\alpha_{j, k}$ and $\alpha_{j, k+1}$, respectively.

We now revisit the error estimates for (3.3) specifically for the range $\theta \in$ $[\pi / 10,9 \pi / 10]$. We start by choosing a parameter $\gamma=0.1562$ in order that $1+8 \gamma^{2}>$ 1.195. Note that for $\theta \in[\pi / 10,9 \pi / 10]$ we have both $\sin (\theta / 2), \cos (\theta / 2) \geq \gamma$.

Next note that in terms of $k$, the contribution of $|P(a, b)|$ from the terms $\{(3,0),(5,0),(7,0),(9,0)\}$ is smaller than

$$
e_{1}=\sum_{n=1}^{4} \frac{2}{(2 n+1)^{2 k+1}} \leq \frac{8}{3^{2 k+1}} .
$$

We now use our new $\theta$-bounds to strengthen the bound on the second error term arising from the set of pairs of the form $(a, a \pm 1)$ with $N \leq 100$. Replacing 0.079 with $\gamma$ in (3.5) yields

$$
|P(1,2)| \leq \frac{2}{1.195^{k}}+\frac{2}{5^{k}}
$$

The contribution from $(3,2),(3,4),(5,4),(5,6),(7,6)$ is smaller than

$$
\frac{10}{1.585^{k}}+\frac{10}{13^{k}} \text {. }
$$

Thus the total contribution from these terms is less than

$$
e_{2}=\frac{2}{1.195^{k}}+\frac{2}{5^{k}}+\frac{10}{1.58^{k}}+\frac{10}{13^{k}} .
$$

We again note that the contribution from the remaining cases with $N \leq 100$ is $e_{3} \leq \frac{44}{9^{k}}$. Similarly, the contribution from terms with $N>100$ is

$$
\begin{aligned}
e_{4} & =\sum_{N=101}^{\infty} 5 N^{1 / 2}((1-C) N)^{-k-1 / 2}<5(1-C)^{-k-1 / 2} \frac{1}{k-1} 100^{-k+1} \\
& <\frac{500}{(k-1) \sqrt{1-C}}(100(1-C))^{-k}<\frac{2283}{(k-1)(4.8)^{k}},
\end{aligned}
$$

where $C=.952$, so that $|\cos (\theta)|<C$ on $(\pi / 10,9 \pi / 10)$.

Putting the error terms all together, we have

$$
\left|R_{2 k+1}\left(z_{\theta}\right)\right|<g(k):=\frac{8}{3^{2 k+1}}+\frac{2}{1.195^{k}}+\frac{2}{5^{k}}+\frac{10}{1.585^{k}}+\frac{10}{13^{k}}+\frac{44}{9^{k}}+\frac{2283}{(k-1)(4.8)^{k}} .
$$

Note that the value of $g(k)$ goes to 0 rapidly when $k$ gets large.

We now return our focus to the relationship between the zeros of $F_{2 k+1}$ and the zeros of $f_{2 k+1}$. Let

$$
h(x)=\frac{2 \pi}{2 x+1}-\frac{2 \pi^{3}}{6(2 x+1)^{3}}-g(x-1) .
$$


Note that $\frac{2 \pi^{3}}{6(2 x+1)^{3}}+g(x-1)$ decays more rapidly than $\frac{\pi}{2 x+1}$; hence, we see that for $k \geq 15$ we have $h(k)>0$. Similarly, note that as $\sin (x) \geq x-\frac{x^{3}}{6}$ for any $0 \leq x<1$, we can conclude that

$$
2 \sin \left(\frac{\pi}{2 k+1}\right) \geq \frac{2 \pi}{2 k+1}-\frac{2 \pi^{3}}{6(2 k+1)^{3}}=h(x)+g(x-1) .
$$

Consider an interval

$$
I_{j, 2 k-1}=\left(\alpha_{j, k}-\frac{2 \pi}{(2 k+1)(2 k-1)}, \alpha_{j, k}+\frac{2 \pi}{(2 k+1)(2 k-1)}\right) \cap[\pi / 10,9 \pi / 10]
$$

about $\alpha_{j, k}$, a zero of $f_{2 k-1}$. We want to show that a zero of $F_{2 k-1}$ lies in this interval. By construction, $I_{j, 2 k-1} \cap I_{i, 2 k+1}=\emptyset$ for any integers $i, j$.

Note that

$$
\begin{aligned}
& i f_{2 k-1}\left(\alpha_{j, k} \pm \frac{2 \pi}{(2 k+1)(2 k-1)}\right) \\
& \quad=2 \sin \left(j \pi \pm \frac{\pi}{2 k+1}\right)=2(-1)^{j} \sin \left( \pm \frac{\pi}{2 k+1}\right) .
\end{aligned}
$$

Hence for even $j$,

$$
\begin{aligned}
i F_{2 k-1}\left(\alpha_{j, k}+\frac{2 \pi}{(2 k+1)(2 k-1)}\right) & =2 \sin \left(\frac{\pi}{2 k+1}\right)+i R_{2 k-1}\left(z_{\alpha_{j}+\frac{2 \pi}{(2 k+1)(2 k-1)}}\right) \\
& \geq 2 \sin \left(\frac{\pi}{2 k+1}\right)-g(k-1) \geq h(k),
\end{aligned}
$$

which is positive. Similarly, $i F_{2 k-1}\left(\alpha_{j}-\frac{2 \pi}{(2 k+1)(2 k-1)}\right) \leq-h(k)$ is negative. Likewise when $j$ is odd, $i F_{2 k-1}$ has opposite signs at the endpoints of $I_{j, 2 k-1}$.

It follows that $F_{2 k-1}$ has an odd number of zeros within $I_{i, 2 k-1}$ as long as it is contained in $[\pi / 10,9 \pi / 10]$. Moreover, since the error term $i R_{2 k-1}\left(z_{\theta}\right)$ is strictly less than the value of $|2 \sin (\theta(2 k+1) / 2)|$ outside of the intervals $I_{j, 2 k-1}$, we can conclude that $F_{2 k-1}\left(z_{\theta}\right)$ has no zero in $[\pi / 10,9 \pi / 10] \backslash \bigcup_{j} I_{j, 2 k-1}$. The analogous result holds for the zeros of $F_{2 k+1}$ with respect to the intervals $I_{j, 2 k+1}$. As in the classical case, we can conclude that within $[\pi / 10,9 \pi / 10]$, the zero intervals $I_{j, 2 k-1}$ for $F_{2 k-1}\left(z_{\theta}\right)$ are separated by the zero intervals $I_{j, 2 k+1}$ for $F_{2 k+1}\left(z_{\theta}\right)$.

As mentioned in Section 1, our method can be generalized to some other families of Eisenstein series, in particular if the zeros of the family under consideration are expected to mainly locate on some fixed hyperbolic line. However, careful analysis is needed to handle the error terms in a more general setting.

\section{ZERos of Eisenstein SERIES AND SPECIAL VALUES OF $L$-SERIES}

We now relate the Eisenstein series $E_{2 k+1, \chi}(z)$ defined by (2.2) to special values of a particular $L$-series by defining

$$
C:=\frac{4(-1)^{k}}{e_{2 k}}=\frac{2(-1)^{k}}{(2 k) ! L(2 k+1, \chi)}\left(\frac{\pi}{2}\right)^{2 k+1} .
$$

We have the following result in the style of (1.1). 
Theorem 4.1. Let $k \geq 1$ be an integer. Then

$$
\frac{2(-1)^{k}}{(2 k) ! L(2 k+1, \chi)}\left(\frac{\pi}{2}\right)^{2 k+1}=4(2 k+1)-16 \sum_{\tau \in \mathbb{H} \backslash \Gamma(2)} \operatorname{ord}_{\tau}\left(E_{2 k+1, \chi}\right) \frac{1}{\lambda(\tau)} .
$$

Proof. We start by building the function

$$
\widetilde{E}_{2 k+1, \chi}(z):=\frac{E_{2 k+1, \chi}(z)}{\Theta_{2}^{4 k}(z) \Theta_{3}^{2}(z)} .
$$

By [LY05, Lemma 2.2], $\Theta_{3}^{2}$ has the same character as $E_{2 k+1, \chi}$; thus $\widetilde{E}_{2 k+1, \chi}$ is a modular function on $\Gamma(2)$. By examining the poles of $\widetilde{E}_{2 k+1, \chi}$ we conclude that it is a monic polynomial in $1 / \lambda$ of degree $k$. We write

$$
\widetilde{E}_{2 k+1, \chi}(z)=\left(\frac{1}{\lambda(z)}-\frac{1}{\lambda\left(\alpha_{1}\right)}\right)\left(\frac{1}{\lambda(z)}-\frac{1}{\lambda\left(\alpha_{2}\right)}\right) \cdots\left(\frac{1}{\lambda(z)}-\frac{1}{\lambda\left(\alpha_{k}\right)}\right) .
$$

Since $\Theta_{2}$ and $\Theta_{3}$ are holomorphic on $\mathbb{H}$, the $\alpha_{j}$ are exactly the zeros of $E_{2 k+1, \chi}$ in $\mathbb{H}$, with multiplicity. Expanding the product in (4.2), we have

$$
\widetilde{E}_{2 k+1, \chi}(z)-\left(\frac{1}{\lambda(z)}\right)^{k}=-\left(\sum_{j=1}^{k} \frac{1}{\lambda\left(\alpha_{j}\right)}\right)\left(\frac{1}{\lambda(z)}\right)^{k-1}+O\left(\frac{1}{\lambda(z)^{k-2}}\right) .
$$

Comparing the coefficients of the $q^{-(k-1) / 2}$, we have

$$
16^{-k}(C-4-8 k)=\frac{-1}{16^{k-1}} \sum_{j=1}^{k} \frac{1}{\lambda\left(\alpha_{j}\right)}=\frac{-1}{16^{k-1}} \sum_{\tau \in \mathbb{H} \backslash \Gamma(2)} \operatorname{ord}_{\tau}\left(E_{2 k+1, \chi}\right) \frac{1}{\lambda(\tau)} .
$$

Solving for $C$ and using (4.1) yield the desired result.

We note that the set of zeros of $G_{2 k+1}(z)=E_{2 k+1, \chi}(z) \mid \gamma_{0}$ is given by

$$
\left\{\tau=\gamma_{0}^{-1} \tau_{0}: \tau_{0} \in \mathbb{H} \backslash \Gamma(2), E_{2 k+1}\left(\tau_{0}\right)=0\right\} .
$$

Therefore, we have the following corollary.

Corollary 4.2. Let $k \geq 1$ be an integer. Then

$$
\frac{2(-1)^{k}}{(2 k) ! L(2 k+1, \chi)}\left(\frac{\pi}{2}\right)^{2 k+1}=4(2 k+1)-16 \sum_{\tau \in \mathbb{H} \backslash \Gamma(2)} \operatorname{ord}_{\tau}\left(G_{2 k+1, \chi}\right) \frac{1}{\lambda\left(\gamma_{0} \tau\right)} .
$$

Proof. This follows directly from Theorem 4.1 and the comment above.

We now consider Eisenstein series of even weight for $\Gamma(2)$. With $S_{k}(\alpha, \beta)$ defined as in (2.1) and $k \geq 2$ an integer, we define

$$
E_{2 k}^{ \pm}(z):=\frac{1}{2}\left(S_{2 k}(0,1) \pm S_{2 k}(1,0)\right) .
$$

These are modular forms of weight $2 k$ for $\Gamma(2)$ with trivial character $\chi_{0}$. In LY05, Long and Yang show that $E_{2 k}^{+}$and $E_{2 k}^{-}$have the Fourier expansions

$$
E_{2 k}^{ \pm}(z)=1 \pm \frac{(2 \pi i)^{2 k}}{4^{k} \Gamma(2 k) L\left(2 k, \chi_{0}\right)} \sum_{r=1}^{\infty} \frac{r^{2 k} q^{r / 2}}{1+\mp(-1)^{r} q^{r / 2}} .
$$


Theorem 4.3. Let $k \geq 2$ be an integer. Then

$$
\frac{(2 \pi i)^{2 k}}{4^{k} \Gamma(2 k) L\left(2 k, \chi_{0}\right)}= \pm 4(2 k)+\mp 16 \sum_{\tau \in \mathbb{H} \backslash \Gamma(2)} \operatorname{ord}_{\tau}\left(E_{2 k}^{ \pm}\right) \frac{1}{\lambda(\tau)} .
$$

Proof. To prove the first result, we set

$$
\widetilde{E}_{2 k}^{+}(z):=\frac{E_{2 k}^{+}(z)}{\Theta_{2}^{4 k}} .
$$

Then $\widetilde{E}_{2 k}^{+}(z)$ is a modular function for $\Gamma(2)$ and is a monic polynomial in $1 / \lambda$ of degree $k$. Arguing as in the proof of Theorem 4.1, we have

$$
\widetilde{E}_{2 k}^{+}(z)-\left(\frac{1}{\lambda(z)}\right)^{k}=-\left(\sum_{\tau \in \mathbb{H} \backslash \Gamma(2)} \operatorname{ord}_{\tau}\left(E_{2 k}^{+}\right)\right)\left(\frac{1}{\lambda(z)}\right)^{k-1}+O\left(\frac{1}{\lambda(z)^{k-2}}\right) .
$$

Equating the coefficients of $q^{-(k-1) / 2}$ on either side, we find that

$$
16^{-k}\left(\frac{(2 \pi i)^{2 k}}{4^{k} \Gamma(2 k) L\left(2 k, \chi_{0}\right)}-8 k\right)=-16^{-(k-1)} \sum_{\tau \in \mathbb{H} \backslash \Gamma(2)} \operatorname{ord}_{\tau}\left(E_{2 k}^{+}\right) .
$$

Solving (4.3) yields the formula for $E_{2 k}^{+}$. The one for $E_{2 k}^{-}$is similarly obtained.

Theorem 4.3 leads directly to the following surprising identity.

Corollary 4.4. Let $k \geq 2$ be an integer. Then

$$
k=\sum_{\tau \in \mathbb{H} \backslash \Gamma(2)}\left(\operatorname{ord}_{\tau}\left(E_{2 k}^{+}\right)+\operatorname{ord}_{\tau}\left(E_{2 k}^{-}\right)\right) \frac{1}{\lambda(\tau)}=\sum_{\tau \in \mathbb{H} \backslash \Gamma(2)} \operatorname{ord}_{\tau}\left(E_{2 k}^{+} E_{2 k}^{-}\right) \frac{1}{\lambda(\tau)} .
$$

\section{REFERENCES}

[DJ08] W. Duke and Paul Jenkins, On the zeros and coefficients of certain weakly holomorphic modular forms, Pure Appl. Math. Q. 4 (2008), no. 4, part 1, 1327-1340. MR2441704

[Gek01] E. U. Gekeler, Some observations on the arithmetic of Eisenstein series for the modular group SL(2, Z), Arch. Math. (Basel) 77 (2001), no. 1, 5-21. MR1845671 (2002f:11050)

[Get04] J. Getz, A generalization of a theorem of Rankin and Swinnerton-Dyer on zeros of modular forms, Proc. Amer. Math. Soc. 132 (2004), no. 8, 2221-2231 (electronic). MR 2052397(2005e:11047)

[Gun06] S. Gun, On the zeros of certain cusp forms, Math. Proc. Cambridge Philos. Soc. 141 (2006), no. 2, 191-195. MR 2265867 (2008d:11035)

[Hah07] H. Hahn, On zeros of Eisenstein series for genus zero Fuchsian groups, Proc. Amer. Math. Soc. 135 (2007), no. 8, 2391-2401 (electronic). MR2302560 (2008a:11047)

[Han58] H. Hancock, Lectures on the theory of elliptic functions: Analysis, Dover Publications Inc., New York, 1958. MR0100106 (20:6540)

[Koh04] W. Kohnen, Zeros of Eisenstein series, Kyushu J. Math. 58 (2004), no. 2, 251-256. MR2117246 (2005h:11084)

[LLY05] W. C. Li, L. Long, and Z. Yang, On Atkin and Swinnerton-Dyer congruence relations, J. Number Theory 113 (2005), no. 1, 117-148. MR2141761(2006c:11053)

[LY05] L. Long and Y. Yang, A short proof of Milne's formulas for sums of integer squares, Int. J. Number Theory 1 (2005), no. 4, 533-551. MR2196794 (2006j:11050)

[MNS07] T. Miezaki, H. Nozaki, and J. Shigezumi, On the zeros of Eisenstein series for $\Gamma_{0}^{*}(2)$ and $\Gamma_{0}^{*}(3)$, J. Math. Soc. Japan 59 (2007), no. 3, 693-706. MR2344823 (2008m:11094)

[Mil02] S. C. Milne, Infinite families of exact sums of squares formulas, Jacobi elliptic functions, continued fractions, and Schur functions, Ramanujan J. 6 (2002), no. 1, 7-149. MR.1906722(2003m:11060) 
[Noz08] H. Nozaki, A separation property of the zeros of Eisenstein series for $\mathrm{SL}(2, \mathbb{Z})$, Bull. Lond. Math. Soc. 40 (2008), no. 1, 26-36. MR2409175 (2009d:11070)

[Ono04] K. Ono, The web of modularity: Arithmetic of the coefficients of modular forms and $q$-series, CBMS Regional Conference Series in Mathematics, vol. 102, published for the Conference Board of the Mathematical Sciences, Washington, DC, by the Amer. Math. Soc., Providence, RI, 2004. MR2020489 (2005c:11053)

[OP04] K. Ono and M. A. Papanikolas, $p$-adic properties of values of the modular $j$-function, Galois theory and modular forms, Dev. Math., vol. 11, Kluwer Acad. Publ., Boston, MA, 2004, pp. 357-365. MR2059773 (2005a:11048)

[RSD70] F. K. C. Rankin and H. P. F. Swinnerton-Dyer, On the zeros of Eisenstein series, Bull. London Math. Soc. 2 (1970), 169-170. MR0260674 (41:5298)

[Shi07] J. Shigezumi, On the zeros of the Eisenstein series for $\Gamma_{0}^{*}(5)$ and $\Gamma_{0}^{*}(7)$, Kyushu J. Math. 61 (2007), no. 2, 527-549. MR 2362897(2008j:11052)

Department of Mathematics, Bucknell University, Lewisburg, Pennsylvania 17837

E-mail address: sharon.garthwaite@bucknell.edu

Department of Mathematics, Iowa State University, Ames, Iowa 50011

E-mail address: linglong@iastate.edu

Department of Mathematics, Oregon State University, Corvallis, Oregon 97301

E-mail address: swisherh@math.oregonstate.edu

Department of Mathematics, Western Washington University, Bellingham, WashingTON 98225

E-mail address: stephanie.treneer@wwu.edu 